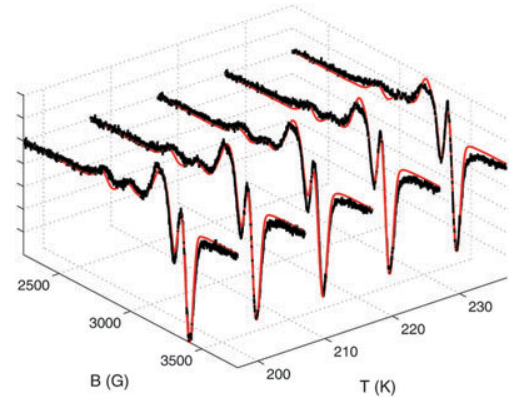

$\mathrm{Cu}($ II)-porphyrin molecular dynamics as seen in a novel EPR/Stochastic Liouville equation study

Pär Håkansson,* ThaoNguyen Nguyen, Prasanth B. Nair, Ruth Edge and Eugen Stulz*

New insights into $\mathrm{Cu}\left({ }_{1}\right)$-porphyrin dynamics quantified using solution EPR experiments and slow-motion line shape simulations.

Please check this proof carefully. Our staff will not read it in detail after you have returned it.

Translation errors between word-processor files and typesetting systems can occur so the whole proof needs to be read. Please pay particular attention to: tabulated material; equations; numerical data; figures and graphics; and references. If you have not already indicated the corresponding author(s) please mark their name(s) with an asterisk. Please e-mail a list of corrections or the PDF with electronic notes attached - do not change the text within the PDF file or send a revised manuscript. Corrections at this stage should be minor and not involve extensive changes. All corrections must be sent at the same time.

Please bear in mind that minor layout improvements, e.g. in line breaking, table widths and graphic placement, are routinely applied to the final version.

Please note that, in the typefaces we use, an italic vee looks like this: $v$, and a Greek nu looks like this: $v$.

We will publish articles on the web as soon as possible after receiving your corrections; no late corrections will be made.

Please return your final corrections, where possible within $\mathbf{4 8}$ hours of receipt, by e-mail to: pccp@rsc.org 


\section{Queries for the attention of the authors}

Journal: PCCP

Paper: c3cp50788b

Title: Cu(II)-porphyrin molecular dynamics as seen in a novel EPR/Stochastic Liouville equation study

Editor's queries are marked on your proof like this Q1, Q2, etc. and for your convenience line numbers are indicated like this $5,10,15, \ldots$

Please ensure that all queries are answered when returning your proof corrections so that publication of your article is not delayed.

\begin{tabular}{|l|l|l|}
\hline $\begin{array}{c}\text { Query } \\
\text { reference }\end{array}$ & \multicolumn{1}{c|}{ Query } & Remarks \\
\hline Q1 & $\begin{array}{l}\text { For your information: You can cite this article before you } \\
\text { receive notification of the page numbers by using the } \\
\text { following format: (authors), Phys. Chem. Chem. Phys., } \\
\text { (year), DOI: 10.1039/c3cp50788b. }\end{array}$ & \\
\hline Q2 & $\begin{array}{l}\text { Please carefully check the spelling of all author names. } \\
\text { This is important for the correct indexing and future } \\
\text { citation of your article. No late corrections can be made. }\end{array}$ & \\
\hline Q3 & $\begin{array}{l}\text { The sentence beginning "For instance, to..." has been } \\
\text { altered for clarity, please check that the meaning is } \\
\text { correct. }\end{array}$ & \\
\hline
\end{tabular}


Cite this: DOI: $10.1039 / \mathrm{c3cp50788b}$

\section{$\mathrm{Cu}(\mathrm{II})$-porphyrin molecular dynamics as seen in a novel EPR/Stochastic Liouville equation study}

\author{
Pär Håkansson, $\dagger^{* a}$ ThaoNguyen Nguyen,$\ddagger^{b}$ Prasanth B. Nair, $\S^{a}$ Ruth Edge $\|^{c}$ and \\ Eugen Stulz*b
}

Received 21st February 2013, Accepted 23rd April 2013 DOI: $10.1039 / \mathrm{c} 3 \mathrm{cp} 50788 \mathrm{~b}$

www.rsc.org/pccp
Copper porphyrin dissolved in $\mathrm{CH}_{2} \mathrm{Cl}_{2}$ :toluene as fluid and frozen solution was studied as a function of temperature using $\mathrm{X}$-band electron paramagnetic resonance (EPR). Quantitative interpretation was obtained using a recently developed Stochastic Liouville simulation method. For the first time we address the large spin system that translates into a 400000 dimensional Liouville equation solved under slowmotion conditions. Using a simple three parameter microscopic model, the temperature dependence of porphyrin rotational correlation time is determined to be in the range 1-10 ns and a fast local motion is in the subpico-second regime with an amplitude increasing with temperature. The methodology provides an important tool for arriving at an accurate set of spin Hamiltonian parameters since determining a unique set of parameters from a frozen solution EPR experiment is often difficult. Thus the proposed method discriminates between parameters proposed from frozen solution EPR experiments or quantum chemistry calculations. The methodology presented is expected to be valuable in obtaining a molecular dynamics picture of metal proteins using EPR as well as in the study of artificial photosynthetic systems.

\section{Introduction}

In studies of environmental effects such as $\mathrm{pH}$ and temperature on transition metal proteins spectroscopic tools such as circudynamic characterisation. ${ }^{1}$ In a general context of biomolecular transition metal complexes, electron paramagnetic resonance (EPR) may provide quantitative information under physiological conditions. From an EPR spectrum of a transition metal dynamics of the metal complex and coordination at the paramagnetic site may be extracted. Hence, there is an opportunity to expand the commonly studied concept of structure $\rightarrow$ functionality to (dynamics and structure) $\rightarrow$ functionality, and

\footnotetext{
${ }^{a}$ School of Engineering Sciences, Computational Engineering and Design Group, University of Southampton, SO17 1BJ, UK. E-mail: p.hakansson@soton.ac.uk

${ }^{b}$ School of Chemistry, University of Southampton, SO17 1BJ, UK.

E-mail:E.Stulz@soton.ac.uk

${ }^{c}$ EPSRC National Service for EPR Spectroscopy, School of Chemistry, The University of Manchester, Manchester, M13 9PL, UK

$\dagger$ Present address: School of Chemistry, University of Southampton, Highfield, SO17 1BJ, UK.

‡ Present address: Reckitt Benckiser, 1st floor, Dansom Lane, Hull, HU8 7DS, UK. $\S$ Present address: University of Toronto Institute for Aerospace Studies, 4925 Dufferin Street, Ontario, Canada M3H 5T6.

55 T Present address: Dalton Cumbrian Facility, The University of Manchester, Westlakes Science \& Technology Park, Moor Row, Cumbria, CA24 3HA, UK.
}

thereby to obtain a detailed microscopic picture. For instance, to perform EPR of a copper protein at physiological temperatures may be critical for evaluating the biological relevance of the experiment and providing a great complement to a frozen solution study. ${ }^{2}$

However, to reach a microscopic understanding of fluidphase biomolecules containing transition metals using EPR experiments, the obstacles to determining spin Hamiltonian parameters as well as accurately computing the EPR line shape have to be overcome. First it is common to determine spin Hamiltonian parameters from EPR experiments with a complex in a frozen solution. Secondly, for copper complexes extra absorption lines (EA) can be expected depending on the frequency of measurement (also referred to as overshoot lines or hyperfine abnormality). ${ }^{3}$ The EA lines impose difficulties in correctly modeling the frozen solution spectra and determining spin Hamiltonian parameters and coordination. ${ }^{4}$ Furthermore freezing artefacts may be present. ${ }^{5,6}$ Thus to verify determined spin Hamiltonian parameters with a carefully analysed fluidphase experiment is much desired. If spin Hamiltonian parameters are instead computed using quantum chemistry methods (QCM) a fluid-phase experimental verification is desirable since QCM for transition metals require a set of approximations to be explored (i.e. solvent, vibrational and relativistic corrections, etc.). ${ }^{7}$ In addition, in order to interpret a fluid-phase EPR experiment, a perturbative treatment ${ }^{8,9}$ is not 
1 valid due to the large anisotropy of Zeeman and transition metal hyperfine parameters: $\Delta g=g_{\|}-g_{\perp}$ and $\Delta A=A_{\|}-A_{\perp}$ are typically 0.15 and $500 \mathrm{MHz}$, respectively, for $\mathrm{Cu}(\mathrm{II})$. Hence the condition for applicable perturbation theory $\left\|\Delta H\left(\Omega_{t}\right)\right\| \tau_{\mathrm{c}} \ll 1$ is not fulfilled, rather $\left\|\Delta H\left(\Omega_{t}\right)\right\| \tau_{\mathrm{c}} \geq 1$ where $\tau_{\mathrm{c}}$ is the characteristic correlation time for slow dynamical modes and $\left\|\Delta H\left(\Omega_{t}\right)\right\|$ is the anisotropy of the spin Hamiltonian. For a metalloprotein, the overall molecular tumbling is slower than $\tau_{\mathrm{c}}$, typically in nanoseconds, and furthermore for anisotropic solvents (such

10 as membranes or liquid crystals) it is expected that slow modes will be present even for small transition metal complexes in a wide range of temperatures, ${ }^{10}$ hence perturbative treatment is not a viable option.

Recently a computational tool to solve the high dimensional

15 Stochastic Liouville Equation in Langevin form (SLEL) was developed. ${ }^{11}$ The SLEL approach enables a non-perturbative simulation of $\mathrm{Cu}(\mathrm{II})$ electron spin coupled to all nearest ligand nuclear spins.

Complications in frozen solution with "extra absorption 20 lines" (EA) have a long history and are discussed by relating spin Hamiltonian parameters to spectral observations for a $\mathrm{Cu}(\mathrm{II})$-porphyrin by Kivelson and Neiman ${ }^{12}$ in 1961 . We refer to cited references for further literature on EA and summarise some key observations here. The number of EA for $\mathrm{Cu}(\mathrm{II})$ complexes is field dependent. ${ }^{4}$ The spectra in the presence of EA cannot be satisfactorily modelled by only assuming a time independent spin Hamiltonian and solving for the relevant energy transitions and finally applying homogenous residual broadening as noted by Kivelson and Neiman. ${ }^{12}$ To proceed, the EPR spectroscopist may then introduce so called strains, i.e. static orientational distributions in the spin Hamiltonian parameters $^{13,14}$ and/or orientation dependent residual line widths. ${ }^{15,16}$ With these additional parameters there may be as many spin Hamiltonian parameters as there are "strain" parameters. ${ }^{4}$ Even with various inhomogeneous linewidths added it is difficult to get a good agreement between simulated and experimental spectra. A better fit is seen in the lower field (L-band). ${ }^{3}$ To establish the number of ligands from EPR spectra it is proposed to analyse only a selected region of spectra, 40 restricting the analysis to a $g_{\perp}$ region $^{4}$ or selecting a $g_{\|}$region of the spectra, ${ }^{17}$ now at a different resonance frequency. It remains an open question if EA is a dynamical effect as opposed to the static picture imposed by strain parameter distributions.

Little work has been done on solving the Stochastic Liouville 45 equation explicitly including ligand nuclear spin for transition metal complexes and thus arriving at a microscopic interpretation of the fluid-phase EPR experiment. For nitroxide spin probes an efficient numerical program for solving the SLE in the frequency domain (EPRLL) was developed by Freed 0 et $a .^{18,19}$ A first complication in extending the work for nitroxide spin probes to transition metals is the large anisotropy of the spin Hamiltonian parameters where the dimension reductions introduced in EPRLL are not valid. The validity problem is seen in work on vanadyl complexes. ${ }^{20,21}$ As a consequence the EPRLL implementation in EasySpin ${ }^{19}$ is not sufficient for $\mathrm{Cu}(\mathrm{II})$. Extending EPRLL and including a complete Liouville spin space is done in a program EPRLF and valid for transition metal complexes, also developed by Freed et $a{ }^{18}$ More recently gadolinium complexes have been studied within the frequency domain approach. ${ }^{22}$ However, the main hurdle comes with the large Liouville space when electron spin couples up to five nuclear spins as is the case for $\mathrm{Cu}(\mathrm{II})$ heme-protein. To proceed, a program has been developed that solves the electron spincopper nucleus SLE problem, ignoring the relaxation channels caused by ligands and then a posteriori convoluting the simulated line shape with the ligand hyperfine splittings. ${ }^{23,24}$ The so called post-convolution method assumes isotropic ligand hyperfine parameters and thus limits possible applicability to copper complexes where this is fulfilled by symmetry or to small complexes where fast molecular tumbling can average these out.

Due to numerical stability issues and memory requirements associated with the solution of high dimensional SLE in the frequency domain ${ }^{24,25}$ a time domain trajectory based method is preferable. ${ }^{11,26-31}$ In this work we show for the first time a multi spin system solved under slow-motion conditions $\left(\left\|\Delta H\left(\Omega_{t}\right)\right\| \tau_{\mathrm{c}} \geq 1\right)$ applied in a line shape study of EPR experiments. The key is the robust implicit numerical scheme developed for solving the SLEL, that conserves the norm of the density matrix and has a known error expansion for combined SLEL and molecular degrees of freedom. ${ }^{11}$ The known error expansion allows for the efficiency of the SLEL simulations to be further improved using higher order interpolation methods. ${ }^{32}$ The numerical scheme allows for very high dimensional Liouville matrices (dimensions 400000 ). Furthermore the SLEL uses time dependent trajectories of the copper complex generated using Brownian dynamics or atomistic molecular dynamics, ${ }^{28,29}$ making SLEL flexible to the dynamical model used, without further increasing the Liouville dimension. Employing SLEL for a complete parameter determination involves significant computational cost and has not yet been attempted to the best of our knowledge. With this in mind, the experimental EPR study is performed in two steps: (i) the frozen solution experiment is used to determine candidate sets of spin Hamiltonian parameters and (ii) the fluid-phase EPR/SLEL study isolates the best set of spin Hamiltonian parameters and determines fluid-phase porphyrin dynamics. In this approach we adopt a back-box view of strain parameters at stage (i) and analysis is carried out using available solid state methods. ${ }^{15}$ The verification stage (ii) benefits from parameters with a microscopic origin, i.e. without ad hoc parameters. We leave for future work the direct determination of EPR parameters using SLEL with a microscopic model of solid state dynamics. To further reduce the computational cost of SLEL simulation, we develop an efficient method for minimisation of the model merit function $\left(\chi^{2}\right)$ to estimate the model parameters. A methodology suitable for computationally expensive simulation methods is introduced which to the best of our knowledge has not been used before in EPR spectroscopy. Briefly, from simulation results evenly spread in the parameter space a training set of $\chi^{2}$ is collected. The training data are used to construct a low cost surrogate model of $\chi^{2}$ which allows for a rapid global fitting procedure. ${ }^{33}$ The present approach significantly 
1 reduces the number of simulations compared to the use of a conventional optimization routine. The $\mathrm{Cu}(\mathrm{II})$-porphyrin system studied has relatively large side chains and by including low temperatures in the fluid-phase experiment (197-293 K) we can expect the slower dynamics, mimicking conditions for large biomolecules.

Here, we present a simple dynamical model that allows for microscopic dynamical information to be extracted. We then show how we can discriminate between different candidate sets of spin Hamiltonian parameters obtained from the frozen solution EPR study and how microscopic insights can be gained from the fluid-phase temperature sequence of experiments.

\section{Theory}

A method for computing a large spin system EPR line shape in SLEL form is outlined in this section and used for interpreting fluid state experiments. The methodology for the solid state line shape can be found in Section 4.1. The following SLEL is numerically solved

$$
\frac{\mathrm{d}}{\mathrm{d} t} \rho\left(\Omega_{t}\right)=-i\left[\mathscr{L}_{\mathrm{S}}+\mathscr{L}_{\mathrm{SL}}\left(\Omega_{t}\right)\right] \rho\left(\Omega_{t}\right),
$$

where $\mathscr{L}_{\mathrm{X}} \equiv\left[H_{\mathrm{X}}, \cdot\right], \mathrm{X}=$ "S", "SL" are superoperators. $H_{\mathrm{S}}$ and $H_{\mathrm{SL}}\left(\Omega_{t}\right)$ are the spin Hamiltonians representing the isolated coherent quantum subsystem $(S)$ and the incoherent spinlattice coupling dependent on a stochastic process in time $\left(\Omega_{t}\right)$, respectively. The spin Hamiltonian contains isotropic and anisotropic components of electron Zeeman, the ligand $30 \quad(N \times 4)$ and copper hyperfine interactions and isotropic copper nuclear Zeeman interaction. $\rho\left(\Omega_{t}\right)$ is a reduced finite-dimensional density operator for electron and nuclear spin degrees of freedom and $i=\sqrt{-1}$. The stochastic time dependence originates in the orientational motions of the copper complex, i.e. relating frames where each type of interaction is defined to the laboratory frame and is simulated explicitly. EPR line shape observable is given by $\overline{\rho_{\text {obs }}\left(\Omega_{t}\right)}=\overline{\operatorname{Tr}\left\{S_{+}^{(1)} \rho\left(\Omega_{t}\right)\right\}}$, where $(\overline{\{.\}})$ denotes the ensemble average. A first derivative EPR line shape is computed as the Fourier-Laplace transform of $\overline{\rho_{\mathrm{obs}}\left(\Omega_{t}\right)}{ }^{29}$

\subsection{A tractable model for $\mathrm{Cu}$ (II) complexes}

To arrive at the model used in SLEL simulation we consider the reference frames of Fig. 1. A principal frame $(\mathrm{P})$ is defined with state it is assumed that the $\mathrm{Cu}(\mathrm{II})$-porphyrin undergoes several internal vibrations and perturbation by solvent causing a fast local averaging of the P-frame. With this in mind, the frame (M) is the average molecular frame over a short time interval and $(\mathrm{L})$ is the laboratory frame defined by the static magnetic field using the spectrometer. $\Omega_{X Y}=\left(\gamma_{X Y}, \beta_{X Y}, \alpha_{X Y}\right)$ are the Euler angles for the $X \rightarrow Y$ transformations.

Thus the angles $\Omega_{\mathrm{MP}}(t)$ are rapidly fluctuating giving the average molecular frame (M). The complete complex is expected to undergo complete rotations in a slower rotational diffusion process, hence the diffusion tensor in Fig. 1. Axial symmetry is
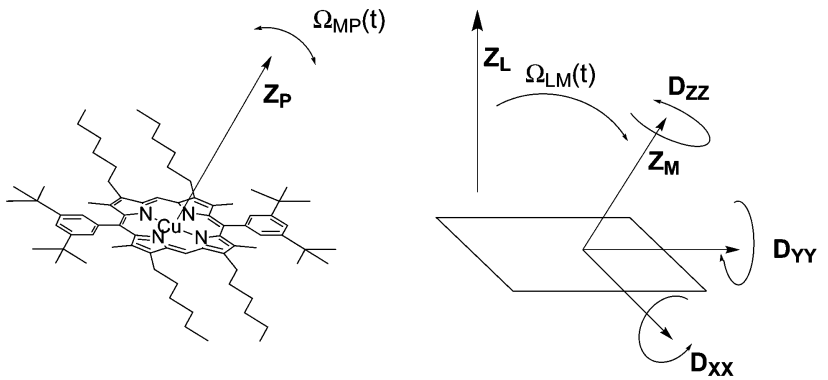

1

Fig. 1 Reference frames: $(P)$ the principal frame along the perpendicular axis relative to the plane of the complex, $(\mathrm{M})$ the molecular reference frame where the diffusion tensor is diagonal and $(L)$ the lab frame defining the static magnetic field.

assumed for Zeeman and hyperfine interactions with the $Z_{\mathrm{P}}$ axis in common.

The Hamiltonian may with these reference frames be divided into a fast fluctuating part (f) and a slowly modulating part (s) as has been found useful in a previous SLEL work. ${ }^{34}$

The following form of the slowly modulated Hamiltonian (indexed with (s)) is derived (see Appendix A.1)

$$
H_{\mathrm{SL}}^{(\mathrm{s})}\left(\Omega_{\mathrm{LM}}(t)\right)=\sum_{\mu, m} A_{\mu, \mathrm{L}}^{(2, m)} D_{m 0}{ }^{2 *}\left(\Omega_{\mathrm{LM}}(t)\right) S^{\mathrm{MP}} F_{\mu, \mathrm{P}}^{(2,0)}
$$

where $A_{\mu, \mathrm{L}}^{(2, m)}$ are the spin operators and $F_{\mu, \mathrm{P}}^{(2,0)}$ are cylindrical symmetric tensor elements both for the interaction $\mu, S^{\mathrm{MP}}=$ $\left\langle D_{00}{ }^{2}\left(\Omega_{\mathrm{MP}}(t)\right)\right\rangle_{\mathrm{MP}}$, is a second rank order parameter averaged over the fast fluctuations of $\Omega_{\mathrm{MP}}(t) . D_{m 0}{ }^{2}\left(\Omega_{\mathrm{LM}}(t)\right)$ are the Wigner rotation matrix elements. ${ }^{35}$ In this context the time dependent part of the Liouvillian takes the form $\mathscr{L}_{\mathrm{SL}}(\Omega(t))=\mathscr{L}_{\mathrm{SL}}^{(\mathrm{s})}\left(\Omega_{\mathrm{LM}}(t)\right)+$ $\mathscr{L}_{\mathrm{SL}}^{(\mathrm{f})}$. The numerical form of eqn (1) is in matrix form where $\mathscr{L}$ is expressed as a static matrix (corresponding to $A_{\mu, \mathrm{L}}^{(l, m)}$ ) times a scalar function $F_{\mu, \mathrm{L}}^{(l, m)}(t)$ with stochastic time dependence (see Appendix A.2). The isotropic $\left({ }^{63} \mathrm{Cu}(\mathrm{II}),{ }^{65} \mathrm{Cu}(\mathrm{II})\right)$ nuclear Zeeman interaction may influence the computed line shape in two ways, a shift of resonance frequency as well as modulating the form of the line shape. However, the influence of shape is found to be negligible with the nuclear Zeeman interaction being 2-3 orders of magnitude less than the rest of the spin Hamiltonian. Hence the $\mathrm{Cu}$ (II) nuclear Zeeman interaction is accounted for as a shift of the resonance frequency computed as the natural abundance average. The fast dynamics is expected to have a small influence on X-band EPR line shape and is in this work accounted for by a relaxation constant $R^{(\mathrm{f})}$ considered to fulfil extreme narrowing conditions. Under this assumption a characteristic correlation time $\left(\tau_{\mathrm{f}}\right)$ of the fast dynamics is available from perturbation theory ${ }^{36}$ (see eqn (A10) in Appendix A.1). In this work we give residual broadening a clear molecular origin in a fast partial average of tensor anisotropy. There are other factors such as uncoordinated solvent protons that are expected to give negligible broadening and thus not included. The approximations underlying eqn (2) are that there is at least a three-fold symmetry in the local fluctuations of $\Omega_{\mathrm{MP}}{ }^{t}$ (see Fig. 1) and a time scale separation between $\Omega_{\mathrm{MP}}{ }^{t}$ and $\Omega_{\mathrm{LM}}{ }^{t}$. With the axial symmetry in magnetic parameters only $D_{X X}$ and $D_{Y Y}$ 
1 modulate the spin Hamiltonian and are assumed to be equal $D_{X X}$ $=D_{Y Y}=D$. The "slow" dynamics is computed explicitly using rotational Brownian dynamics trajectories providing $\Omega_{\mathrm{LM}}{ }^{t} \cdot{ }^{37}$ The parameters entering SLEL simulation related to dynamics are $\left\{D, S^{\mathrm{MP}}, R^{(\mathrm{f})}\right\}$. The implicit numerical scheme developed for SLEL problems in ref. 11 enables us to tackle this large spin system.

\section{Material and methods}

The sample consists of copper(II) di-tert-butyl diphenyl porphyrin (CuDPP) with solvent in a dichloromethane:toluene $(10: 1)$ mixture. The di-tert-butyl diphenyl porphyrin synthesis has been described previously. ${ }^{38}$ Metallation was performed by adding a suspension of copper(II) acetate monohydrate (247 $\mathrm{mg}, 1.36 \mathrm{mmol}$ ) in acetic acid $(10 \mathrm{~mL})$ to a solution of the porphyrin $(49.0 \mathrm{mg}, 45.4 \mu \mathrm{mol})$. The reaction mixture was heated for $3 \mathrm{~min}$, then diluted with dichloromethane $(30 \mathrm{~mL})$. The organic layer was washed with water $(6 \times 50 \mathrm{~mL})$, followed by drying over $\mathrm{Na}_{2} \mathrm{SO}_{4}$, filtered through cotton wool and concentrated in vacuo to give the CuDPP $(67.0 \mathrm{mg}$, quantitative) as purple solid (LRMS [MALDI-ToF]: calcd $m / z$ for $\mathrm{C}_{76} \mathrm{H}_{108} \mathrm{~N}_{4} \mathrm{Cu}^{+}$ $[\mathrm{M}]^{+}$1139.8, found 1139.8).

Frozen solution first derivative spectra were recorded at $120 \mathrm{~K}$, Modulation Amplitude $2 \mathrm{G}$ with one scan, microwave frequency $9.4391 \mathrm{GHZ}$, power $3.991 \mathrm{~mW}$, $84 \mathrm{~s}$ sweep time and 2048 points. Fluid state spectra were recorded at thirteen temperatures between $197 \mathrm{~K}$ and $293 \mathrm{~K}$ at $8 \mathrm{~K}$ intervals, modulation amplitude $5 \mathrm{G}$ and resonance around $9.4 \mathrm{GHz}$ using four scans. The temperature was monitored using a thermocouple situated just below the sample. To verify the minimum $20 \mathrm{~min}$ temperature equilibration, a cycle of temperatures were performed to verify that spectra could be overlaid.

\subsection{SLEL computational details}

In this section a SLEL simulation is exemplified and we discuss how to distribute computational efforts in an efficient manner. In Fig. 2 the simulated free induction decays (FIDs) (imaginary part) and the corresponding first derivative EPR line shape are fumber for a relatively slowly tumb case $\left\|\Delta H\left(\Omega_{t}\right)\right\| \tau_{\mathrm{c}}=6$ The displayed result is the powder averaged SLEL simulation with the 419904 dimensional Liouville matrix with the density matrix simulated up to $72 \mathrm{~ns}$. It is worth noting that the simulation is straight forward to divide into several parallel each simulating initial molecular frame in a specific initial powder angle segment. The parallel jobs exploit a computer cluster and scales linearly. The memory requirement for one SLEL simulation is around 3.5 Giga bytes, enabling the simulation to be carried out using the low memory nodes on lation is completed in 40-60 hours if $3002.27 \mathrm{GHZ}$ processors are employed, averaging over a total of 10000 trajectories. Note further from Fig. 2 that a large part of $\operatorname{FID}(t)$ has a low amplitude. These low amplitude oscillations need to be included in particular to account for partially resolved ligand hyper fine splittings in the high field end of line shape (around 3400 Gauss in Fig. 2).
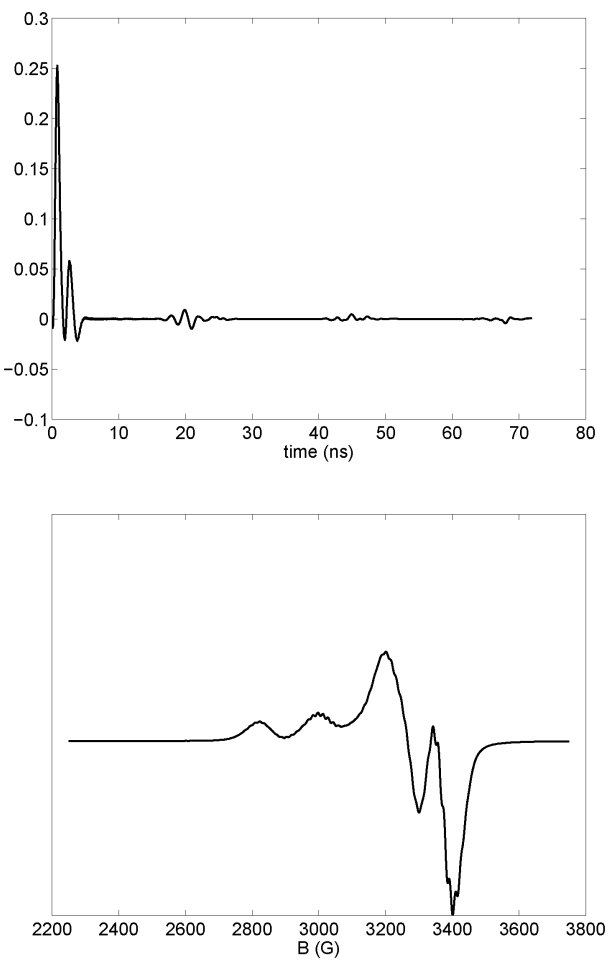

Fig. 2 SLEL simulation: imaginary part of $\operatorname{FID}(t)$ and first derivative line shapes in top and bottom panels respectively. Parameters $D=3 \times 10^{7} \mathrm{~s}^{-1}, \mathrm{~S}^{\mathrm{MP}}=0.9, R^{(\mathrm{f})}=$ $3.2 \mathrm{G}$ and spin Hamiltonian parameters $\mathrm{A}$ in Table 1 corresponding to a dynamical regime $\left\|\Delta H\left(\Omega_{t}\right)\right\| \tau_{c}=6$. The result is the ensemble average over 8000 trajectories, corresponding to 42 hours simulation time employing 300 processors.

However, these are unresolved at low temperatures as seen from the experimental spectra below.

In this work we address the large SLEL computational task in two ways. First, since the full length simulation is large, we divide the analysis of liquid state experiments into two stages, where first the spectra are analysed using simulations truncated at 7.6 ns, referred to as T-SLEL. The T-SLEL has a factor of 10 shorter time span and requires 4000 trajectories for smooth line shape and hence is a factor of 20 faster to complete. This serves to obtain a first estimate of the parameter space $\left\{D, S^{\mathrm{MP}}\right\}$. As seen from Fig. 3, the T-SLEL form captures the main features

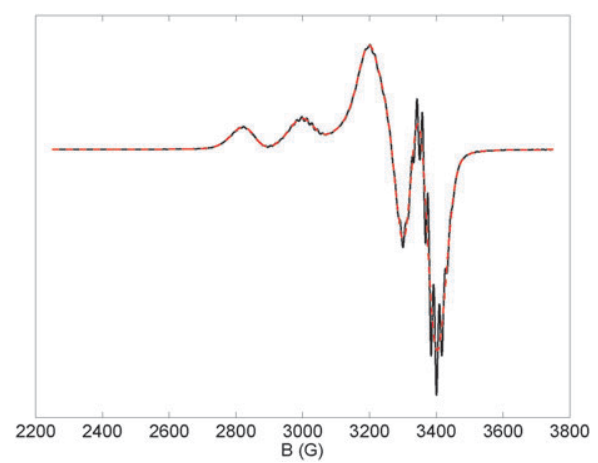

Fig. 3 First derivative line shape computed from 72 ns SLEL (solid black line) and T-SLEL $7.2 \mathrm{~ns}$ (red dashed line). Parameters, $D=3 \times 10^{7} \mathrm{~s}^{-1}, \mathrm{~S}^{\mathrm{MP}}=0.9$ and $R^{(\mathrm{f})}=0.9 \mathrm{G}$. The $R^{(\mathrm{f})}$ is particularly low to illustrate ligand hyperfine splitting at the high field end of spectra. 
and is a useful method to model broad unresolved spectra seen at lower temperatures.

Secondly, parameter estimation is addressed in an efficient and robust manner as described below. Throughout this work the quality of the fit is quantified with the following model merit function,

$$
\hat{\chi}^{2}\left(B_{i}\right)=\frac{1}{N} \sum_{k=1}^{i} \frac{\left[\left(I^{\prime}\left(B_{k}\right)\right)_{\operatorname{Exp}}-\left(I^{\prime}\left(B_{k}\right)\right)_{\mathrm{Mod}}\right]^{2}}{\sigma^{2}},
$$

where $N$ is the number of experimental points, $B_{k}$ denotes the field strength $I^{\prime}\left(B_{k}\right)_{\mathrm{X}}, \mathrm{X}=\mathrm{Exp}$, Mod are first derivative spectra of the experiment and the model, respectively, and $\sigma^{2}$ is the experimental variance estimated from the low field tail of spectra.

The function $\chi^{2}$ is a challenge to minimize for several reasons: it is noisy, containing experimental as well as modelling uncertainties and it may have several minima. In an overview of minimizing $\chi^{2}$ in the context of slow-motion EPR, ${ }^{39}$ it is recognised that $\chi^{2}$ is noisy and for this reason the "Nelder-Mead simplex" is the better compared to Newton and Levenberg-Marquardt algorithms. The advantage of the "Nelder-Mead simplex" is that the derivatives of $\chi^{2}$ are not needed. ${ }^{39}$ Both "Nelder-Mead simplex" and LevenbergMarquardt algorithms may be successfully integrated with the so called simulating annealing method to avoid getting stuck in local minima. ${ }^{40}$ However, in this work our goal is to minimize the total number of function evaluations, which are inherent in above methods as well as in genetic algorithm based global search techniques. ${ }^{41}$ For this reason we work with an approximation model of $\chi^{2}$ that is computationally cheap to evaluate (also referred to as a surrogate model). In particular, we employ a Gaussian process (GP) regression model ${ }^{33}$ based optimization strategy that involves the following iterative steps: (i) sampling of a training set $\hat{\chi}^{2} \in \mathbb{R}^{K}$ at $K$ uniformly selected fitting parameter sets $(x)$ on a bounded domain, (ii) construction of a GP model $\left(\chi^{*}\right)^{2}$ as a function of the fitting parameters, (iii) global minimisation of $\left(\chi^{*}\right)^{2}$ and finally (iv) update the training set $\hat{\chi}^{2}$ with the current best fit and return to step (ii) or stop if an acceptable fit has been found.

The initial training set in step (i) is constructed from typically 15 simulations with $x=\left\{D, S^{\mathrm{MP}}, R^{(\mathrm{f})}\right\}$ distributed over the domain $(0.4 \leq D \leq 80) \times 10^{7} \mathrm{~s}^{-1}, 0.65 \leq S^{\mathrm{MP}} \leq 1$ and $2 \leq R^{(\mathrm{f})} \leq 50 \mathrm{G}$. Each simulation result is post processed with $R^{(\mathrm{f})}$. After minimisation, the training set typically consists of 35 individual simulations (with various values of $\left\{D, S^{\mathrm{MP}}\right\}$ ) and a total of 900 training points after processing with $R^{(\mathrm{f})}$. The simulated line shapes and experimental spectra are both normalised to unit area with $I^{\prime}(B) / \int\left|I^{\prime}(B)\right| \mathrm{d} B$ before $\hat{\chi}^{2}\left(B_{N}\right)$ is computed (eqn (3)), with the list of parameters and corresponding $\hat{\chi}^{2}$ making up the training set. The task in step (ii) involves constructing a computationally cheap GP surrogate $\left(\chi^{*}\right)^{2}$ replacing $\chi^{2}$, such that all of our knowledge of $\chi^{2}$ is exploited. The value of $\left(\chi^{*}\right)^{2}$ at the parameter set $x^{*}$ can be obtained from

$$
\left[\begin{array}{c}
\hat{\chi}_{1}^{2} \\
\vdots \\
\left(\chi^{*}\right)^{2}
\end{array}\right] \sim \mathscr{N}\left[1 \beta,\left(\begin{array}{ccc}
\Gamma\left(x^{1}, x^{1}\right), \Gamma\left(x^{1}, x^{2}\right) & \cdots & \Gamma\left(x^{1}, x^{*}\right) \\
\vdots & \ddots & \vdots \\
\Gamma\left(x^{*}, x^{1}\right), \Gamma\left(x^{*}, x^{2}\right) & \cdots & \Gamma\left(x^{*}, x^{*}\right)
\end{array}\right)\right]
$$

where the dimensionality of $\hat{\chi}$ is $K,\left(\chi^{*}\right)^{2}$ is a multivariate Gaussian, $\beta$ is the mean, $\mathbf{1}$ is a $(K+1)$ vector of ones, $\Gamma\left(x, x^{*}\right)=$ $\hat{\sigma}^{2} \prod_{i}^{3} \exp \left(-\theta_{i}\left|x_{i}-x_{i}^{*}\right|^{2}\right)$ is the covariance function used in GP modeling. The hyperparameters of the GP model $\left(\beta, \hat{\sigma}, \theta_{i}\right)$ are determined from the training dataset using a maximum likelihood estimation procedure. Two forms of uncertainty in the GP model for $\left(\chi^{*}\right)^{2}$ can be identified: (a) the GP model is constructed from a finite set of simulations and (b) each simulation result in $\hat{\chi}^{2}$ contains uncertainty originating from experimental noise as well as a small amount of simulation error (i.e. arising from the use of a finite set of trajectories). The uncertainty (a) is accounted for in an inherent way with the use of a Gaussian process model. In this work we address uncertainty (b) by performing a regression of training data by including a hyperparameter for noise in the GP covariance function. ${ }^{33}$ In this way an account of uncertainty in $\hat{\chi}^{2}$ is taken as opposed to the case when training data are interpolated and $\hat{\chi}^{2}$ are treated as noise-free values. A global minimisation (step (iii)) is rapidly performed using a standard minimisation routine ${ }^{39}$ here the global search is done with simulated annealing. ${ }^{33}$ Finally, optimisation is terminated manually in step (iv) when $\left(\chi^{*}\right)_{\min }{ }^{2}$ is similar to updated training set $\hat{\chi}_{\min }{ }^{2}$. With the cost being large for each simulation, the GP model $\left(\chi^{*}\right)^{2}$ is a valuable compromise making the best of the information available $\left(\hat{\chi}^{2}\right)$ in a Bayesian statistics sense. Moreover, the task when using conventional minimization routines to compute the gradient and hessian of a noisy function is completely avoided.

\section{Results and discussion}

The main focus of this study is to explore the fluid state EPR experiment (197-293 K) using the SLEL simulation method. The two main questions addressed are: can a discrimination be made between three given spin Hamiltonian parameter sets and determine the one in closest agreement with experiment? Secondly, can we quantify the $\mathrm{Cu}(\mathrm{II})$-porphyrin dynamics at different temperatures? First, determination of spin Hamiltonian sets from frozen solution is discussed with subsequent presentation of the fluid state results.

\subsection{K EPR experiment}

The frozen solution EPR experiment is analysed using the solid state line shape function (pepper) in EasySpin. ${ }^{15}$ The pepper function allows for a computed powder average using second order perturbation theory for ${ }^{14} \mathrm{~N}$ ligands. The spin Hamiltonian in pepper includes electron Zeeman, the ${ }^{63} \mathrm{Cu}$ (II) and ${ }^{65} \mathrm{Cu}$ (II) isotropic nuclear Zeeman (weighted at a natural abundance ratio), $\mathrm{Cu}(\mathrm{II})$ hyperfine and four ${ }^{14} \mathrm{~N}$ ligand hyperfine interactions. The routine pepper provides line broadening to mimic a frozen solution spectrum. Anisotropic parameters have a cylindrical symmetry as is expected for a square planar complex. In this work the inhomogeneous broadening ${ }^{15}$ of the so called Hstrain, gstrain and Astrain form are explored. 
1 Table 1 Minimization result for $X$-band $120 \mathrm{~K}$ experiments. $\sigma_{R}$ denotes isotropic broadening, $\sigma_{X}, \sigma_{X}$ for $X=H, g, A$, denotes directional components of Gaussian line width at half height, and Gaussian distribution width of $g$ and $A$ parameters respectively. Units for all magnetic parameters are in Gauss. $\chi^{2}$ [cf. eqn (3)] is computed over the whole spectra for data sets $\{A, B\}$ and range (2550-3100

5 Gauss) for $C$

\begin{tabular}{lllllll}
\hline \multicolumn{7}{c}{ Hamiltonian parameters } \\
\cline { 2 - 7 } Data set & $g_{\perp}$ & $g_{\|}$ & $A_{\perp}$ & $A_{\|}$ & $A_{\perp}{ }^{N}$ & $A_{\|}{ }^{N}$ \\
\hline A & 2.045 & 2.191 & 20.57 & 211.93 & 17.11 & 13.76 \\
C & 2.011 & 2.187 & 15.68 & 209.72 & 18.92 & 16.64 \\
B & 2.056 & 2.187 & 20.56 & 208.58 & 17.14 & 15.49 \\
\hline
\end{tabular}

Phenomenological parameters

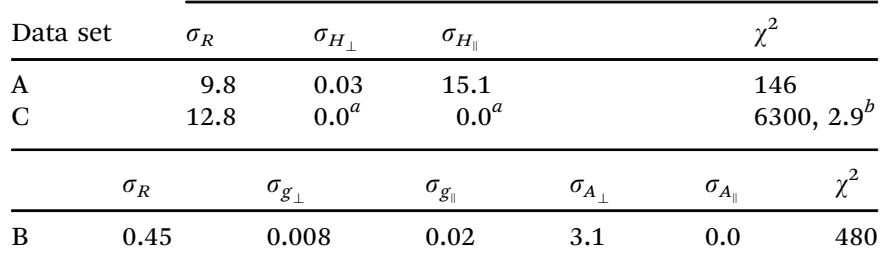

${ }^{a}$ Held fixed during minimization. ${ }^{b} \chi^{2}$ computed over 2550-3100 Gauss.

Minimization of eqn (3) is done with the matlab function fminsearch, using the Nelder-Mead simplex algorithm. ${ }^{42}$ Table 1 gives the best fit parameters.

Three approaches are tested concerning broadening of frozen solution spectra resulting in the data sets denoted by A, B and C in Table 1. All three data sets have, except spin Hamiltonian parameters, a homogeneous $\left(\sigma_{R}\right)$ broadening term. The data set A has, in addition, orientation dependent line width terms $\left(\sigma_{H_{\perp}}\right.$ and $\left.\sigma_{H_{\|}}\right)$and the data set B has four gStrain and AStrain terms with perfectly correlated and static $g_{-}$ $A$ frame distributions. For data sets A and B we searched a best fit using the whole spectra whereas for data set $\mathrm{C}$ the best fit was sought in the low field part (2550-3100) G. A motivation for only using low field part in data set $\mathrm{C}$ is that only a single homogeneous broadening constant is needed to obtain a low $\chi^{2}$ value and this region is less complicated by EA phenomena. The idea to analyse only a section of copper EPR spectra is used in several works. ${ }^{4}$ The $120 \mathrm{~K}$ EPR experiment and simulated line shapes are provided in the upper panel of Fig. 4, together with the cumulative deviation for each simulation in the lower panel. Note that the best fit concerning the whole spectral width is data set A. However concerning only the low field region, where the problem with EA is less pronounced, the data sets B and C are slightly better. This motivates us to test all three data sets using the above SLEL liquid state study.

\subsection{Liquid state EPR experiments}

Given the large computational cost in completing a full SLEL simulation, a suitable parameter space for the liquid state experiments is first estimated using truncated simulations T-SLEL after which full length SLEL simulations are used.

For the spin Hamiltonian parameter set A in Table 1, the estimated parameters and line shapes are given in Table 2 and Fig. 5. The experiments (black solid lines in Fig. 5) show
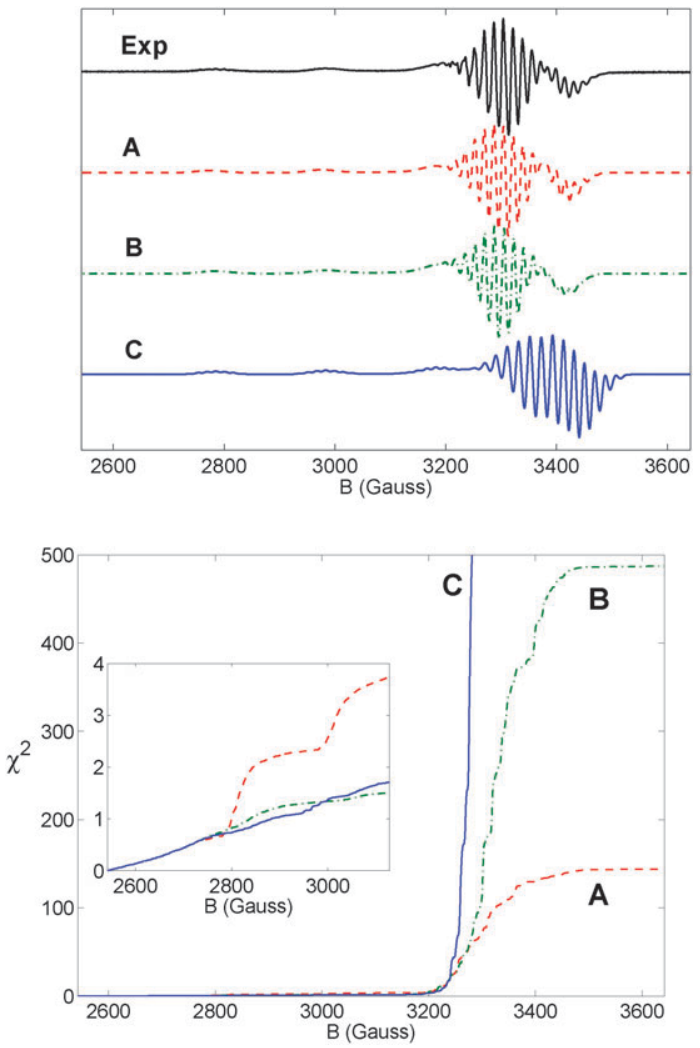

Fig. 4 The top panel displays the $120 \mathrm{~K}$ X-band experiment followed by simulations using spin Hamiltonian data sets $A, B$ and $C$ respectively. The lower panel displays cumulative deviation sum $\chi^{2}\left(B_{i}\right)$ [cf. eqn (3)] with a lower field part inserted.

increasingly resolved hyperfine splitting with temperature. Furthermore spectra remain relatively broad even at $293 \mathrm{~K}$, not reaching the Lorentizian shapes characteristic of a complex in the fast tumbling regime. From Table 2 it is seen from $\hat{\chi}^{2}$ that the T-SLEL agrees better in the low temperature region and gradually becomes worse at higher temperature. The upper theoretical limit for $S^{\mathrm{MP}}$ is one, hence found parameters suggest that melting the sample has reduced the order if we assume order 1 for frozen solution.

Since ligand hyperfine couplings contribute to the broadening of the spectra but a full length simulation is need to account for any resolved ligand hyperfine splitting, we return to the high field discrepancy in Section 4.3 and also explain the constant $D$ versus temperature for the higher temperatures in Table 2.

For spin Hamiltonians in sets B and C [cf. Table 1], optimal fits are sought only in the low temperature region. In Fig. 6 we show best fit T-SLEL line shapes fluid state $197 \mathrm{~K}$ together with spectra and merit function $\chi^{2}\left(B_{i}\right)$ (eqn (3)) in the left and right panel respectively. For set B the best fit parameters are: $\{D=6.6$ $\left.\times 10^{7} \mathrm{~s}^{-1}, S^{\mathrm{MP}}=1, R^{(\mathrm{f})}=13 \mathrm{G}\right\}$ and for set $\mathrm{C}\left\{D=30 \times 10^{7} \mathrm{~s}^{-1}\right.$, $\left.S^{\mathrm{MP}}=1, R^{(\mathrm{f})}=46 \mathrm{G}\right\}$. Note from Fig. 6 (inset) that, for the data set $\mathrm{C}$, although $\chi^{2}$ is low in the lower field region the total $\chi^{2}$ is large. Although both data sets B and A have low $\chi^{2}$ (see Fig. 6) there is no possibility of further improving the fit for B within 
1 Table 2 T-SLEL data set used in Fig. 5 for liquid state EPR experiments. Spin Hamiltonian parameters are from the set $A$ in Table 1. From left, temperature, diffusion constant, local order parameter, residual line width and $\hat{\chi}^{2}\left(B_{N}\right)$

\begin{tabular}{|c|c|c|c|c|}
\hline$T / \mathrm{K}$ & $D / 10^{7} \mathrm{~s}^{-1}$ & $S^{\mathrm{MP}}$ & $R^{(\mathrm{f})} / \mathrm{G}$ & $\hat{\chi}^{2}$ \\
\hline 197 & 1.5 & 0.90 & 18 & 5.0 \\
\hline 205 & 3.0 & 0.90 & 20 & 8.6 \\
\hline 213 & 8.0 & 0.90 & 20 & 11.5 \\
\hline 221 & 9.0 & 0.80 & 22 & 9.7 \\
\hline 229 & 9.0 & 0.80 & 17 & 19.7 \\
\hline 237 & 9.0 & 0.80 & 18 & 9.7 \\
\hline 245 & 9.0 & 0.75 & 13 & 28.3 \\
\hline 253 & 9.0 & 0.75 & 10 & 14.9 \\
\hline 261 & 9.0 & 0.70 & 10 & 11 \\
\hline 269 & 9.0 & 0.70 & 9.5 & 378 \\
\hline 277 & 9.0 & 0.65 & 10 & 381 \\
\hline 285 & 9.0 & 0.65 & 9.1 & 463 \\
\hline 293 & 9.0 & 0.65 & 9.1 & 672 \\
\hline
\end{tabular}

the three parameter dynamical model. For instance the line shape needs to be broader, however $S^{\mathrm{MP}}$ is already at its maximum value and a better fit is not achieved with a slower rotational diffusion. Hence the best fit is found for spin Hamiltonian set A with a factor of two lower total $\chi^{2}$ as compared to B. The discrimination between spin Hamiltonian sets may continue using full length SLEL simulations, however we find that at low temperature the difference is small between SLEL and T-SLEL (see Fig. 3), and full length SLEL simulations are not needed in order to conclude $\mathrm{A}$ as the best candidate.

Note that within the proposed microscopic model we thus discriminate between two spin Hamiltonian parameters (A and B) only differing within a few percent, not relying on phenomenological parameters. Low temperature EPR experiments are particularly valuable since dynamics is in a semi-static regime. In this regime, EPR is clearly sensitive to dynamics, however anisotropic diffusion is expected to have small effect and thus we can focus on two dynamic parameters $\left\{D, S^{\mathrm{MP}}\right\}$. For higher

35 temperatures an anisotropic diffusion model may be needed as is seen from nitroxide spin probe studies. ${ }^{18}$ Such a model is already implemented, however, it adds an unnecessary complexity to the task of finding the best candidate spin Hamiltonian parameters from low temperature fluid state EPR experiments.

\subsection{Detailed fluid solution study}

For the T-SLEL study, the quality of the fit is reduced at the higher temperatures (Table 2, Fig. 5). However, the information is still a valuable starting point in performing full length SLEL simulations. To obtain a detailed picture of the dynamics as well as to explore what insights the full length simulated SLEL gives, the results for temperatures 205, 253 and $277 \mathrm{~K}$ are provided in Table 3 and Fig. 7. For temperatures $205 \mathrm{~K}$ and the $277 \mathrm{~K}$ experiment, parameters restricted to the intervals $(3<D<20) \times 10^{7} \mathrm{~s}^{-1}$ and $0.65<S^{\mathrm{MP}}<1$ are re-optimised. $R^{(\mathrm{f})}$ is manually adjusted for all three temperatures to best explain the ligand hyperfine splittings. From Fig. 7 it is seen that full length SLEL simulation accounts for main features of the temperature sequence of experimental spectra. The model

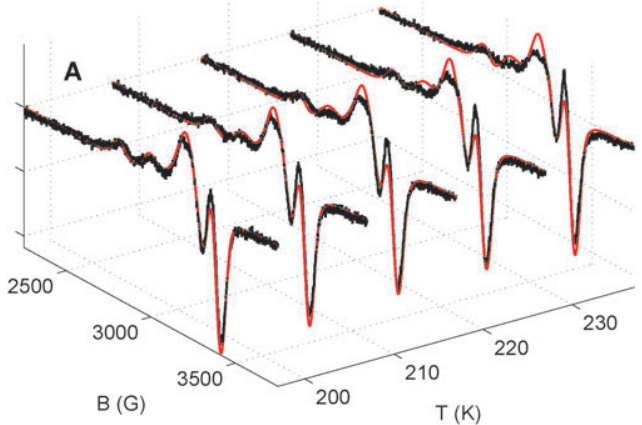
. . .7 (n) , 
Fig. 6 The top panel displays the $X$ band experiment at $197 \mathrm{~K}$ (black solid lines) and the simulated T-SLEL line shape (truncated at $7.6 \mathrm{ns)} \mathrm{using} \mathrm{spin} \mathrm{Hamiltonian}$ parameter sets A (red dashed line), B (black dot-dashed line) and C (blue solid line) respectively. The bottom panel displays model merit function $\chi^{2}\left(B_{i}\right)$ (eqn (3))

Table 3 SLEL data set used in Fig. 7 for liquid state EPR experiments. Spin Hamiltonian parameters are the set $A$ in Table 1. From left, temperature, diffusion constant, local order parameter, line broadening, $\tau_{f}$ calculated using eqn (A10) and $\chi^{2}$ [cf. eqn (3)]. Overall rotational correlation time is given by $\tau_{c}=1 / 6 D$

SLEL

\begin{tabular}{rrlllr} 
& \multicolumn{2}{l}{ SLEL } & & & \\
\cline { 2 - 5 }$T / \mathrm{K}$ & $D / 10^{7} \mathrm{~s}^{-1}$ & $S^{\mathrm{MP}}$ & $R^{(\mathrm{f})} / \mathrm{G}$ & $\tau_{\mathrm{f}} / \mathrm{ps}$ & $\chi^{2}$ \\
\hline 205 & $3.0^{a}$ & $0.9^{a}$ & 12.9 & 1.7 & 13 \\
253 & $9.0^{a}$ & $0.8^{a}$ & 4.2 & 0.30 & 19 \\
277 & $14.9^{b}$ & $0.68^{b}$ & 2.9 & 0.13 & 357
\end{tabular}

${ }^{a}$ From Table 2. ${ }^{b}$ Optimized.

model a limited averaging of the signal is explained in terms of

copper-phthalocyanine molecules that undergo a complicated rotational motion"44 (pages 1945-1946). However, in these works either a perturbative line shape or a post convolution Stochastic Liouville method m,24,44 $^{20}$ used. Such approaches suffer from theoretical limitations; for instance, the post convolution approach lacks theoretical foundation for non-zero ligand hyperfine anisotropy. Hence, caution must be exercised while applying these methods in order to avoid erroneous conclusions.

We comment on the unexpected trend in Table 2 with first increasing and then constant rotational diffusion constant $(D)$ versus temperature. We find that the fit is improved using full length simulations, illustrated with the experiment at $277 \mathrm{~K}$ in

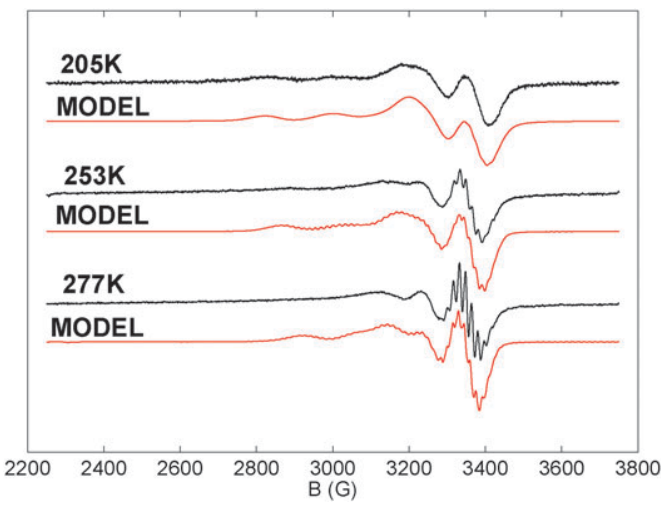

Fig. $7 \quad X$ band experimental spectra (black solid lines) and simulated line shapes (red solid lines) for temperatures 205, 253 and $277 \mathrm{~K}$.

Table 3. A larger, physically more realistic rotational diffusion constant is found compared to Table 2, suggesting that analyzing data with full length simulation is needed for higher temperatures. The porphyrin dynamics accounted for include two dynamical modes: a local fluctuation and an overall molecular tumbling. With increasing temperature, both $S^{\mathrm{MP}}$ and $\tau^{(\mathrm{f})}$ decrease, corresponding higher energy local modes being populated, seen in the increasing amplitude and rate of local motion (Table 3). The amplitude of local motion is a parameter describing (dynamic) deformations of the porphyrin structure. The porphyrin deformations are suggested to have biological relevance $^{45}$ and large dependence on the type of peripherally attached groups and porphyrin metal. ${ }^{46}$ The presented EPR/ SLEL methodology provides quantitative experimental data on this aspect. An interesting continuation of this work may involve an anisotropic rotational model or an all-atomistic molecular dynamics EPR/SLEL study that may well improve the fit for higher temperatures as well as provide insight into the dynamics of porphyrin deformations for different peripherally attached groups.

Clearly the SLEL calculations can be refined further, for instance one way forward is to go beyond a constant $\left(R^{(\mathrm{f})}\right)$ broadening and use the full matrix form as outlined in Appendix A.1. The spin Hamiltonian can be extended with components such as spin rotation ${ }^{6}$ that may have a small contribution that can be included in $\mathscr{R}^{(\mathrm{f})}\left(\Omega_{\mathrm{LM}}(t)\right)$ (see eqn (A8) in Appendix A.1). These extensions are readily available, do not further increase memory requirement and may improve the fit. This is outside the scope of the present work and is left for future studies.

\section{Summary}

In 1982 Hyde and Froncisz ${ }^{6}$ estimated that a molecular weight of 1000 is the upper limit at which information about rotational tumbling rates of copper transition metal complexes at room temperature may be extracted using perturbation theory. This mass limit rules out studies on the majority of biologically relevant copper complexes even if spin Hamiltonian parameters are completely known. Very little has been done in the EPR community to overcome this limitation. For instance the use of a Stochastic Liouville equation to simulate a copper 

hyperfine splittings assumes that the anisotropy of ligand hyperfine parameters is averaged to zero, an assumption that is not valid for slowly tumbling complexes.

In this work we present for the first time, simulations of SLEL line shapes for the complete spin system, that provides the EPR community with a tool to verify proposed spin Hamiltonian parameters and extract rotational correlation times as well as amplitudes and rates of fast restricted local motion.

We have concluded on a best set of spin Hamiltonian parameters proposed from a frozen solution EPR study (see A in Table 1), which also shows consistency with a fluid solution study. The ability to verify with the fluid solution EPR study is critical since the frozen solution study may be hampered by EA lines and possibly freezing artefacts. ${ }^{6}$ We find exceptionally good fits to low temperature fluid EPR experiments using a simple dynamical model. This is contrary to previous slow-motion studies where low temperature shows largest model discrepancies for the two spin system, ${ }^{20}$ or the six spin system using the post convolution method. ${ }^{24}$ Using a full length SLEL simulation we conclude on the dynamics of copper-porphyrin with an overall tumbling in the regime $\tau_{\mathrm{c}}=1-10 \mathrm{~ns}$ and a subpico-second fast restricted local motion with amplitude increasing with temperature (see Table 3). The fast local motion is found to play an important role in the resolved ligand hyperfine lines at higher temperature X-band experiments. A Gaussian process regression is found to simplify and reduce the computational cost when fitting line shapes, and is important when using high cost simulation methods to analyse experiments. The number of unknown parameters may be reduced with the use of molecular dynamics simulations (MD) where a short MD simulation may be used to compute local order parameters, even for larger biomolecules. With a reliable force field for copper complexes, the SLEL may be simulated using MD trajectories directly. ${ }^{29}$ The SLEL allows us to address low filed magnetic resonance experiments even if the Liouville matrix dimension is relatively large. This is not possible with perturbative relaxation theory and is particularly important in the exploration of MRI relaxation agents. ${ }^{47}$

With a detailed account of transition metal dynamics made available by EPR and SLEL, several interesting questions arise. For instance, energy transfer in artificial photosynthetic systems $^{48}$ may have a dependence on local or tumbling dynamics and EPR/SLEL can improve the design of these systems. Similarly in homogeneous catalysis, ${ }^{49}$ dynamics of a efficiency and EPR/SLEL may again be used to improve molecular design.

The simulation program developed during this research will be made available with an extensive set of tools under the GNU open source licence.

\section{A.1}

The spin Hamiltonian is in a generally applicable format expressed in spherical tensor notation as follows: ${ }^{50}$

$$
\begin{gathered}
H\left(\Omega_{\mathrm{LM}}{ }^{t}, \Omega_{\mathrm{MP}}{ }^{t}\right)=H_{\mathrm{S}}+H_{\mathrm{SL}}\left(\Omega_{\mathrm{LM}}{ }^{t}, \Omega_{\mathrm{MP}}{ }^{t}\right) \\
H_{\mathrm{S}}=\sum_{\mu} F_{\mu}^{(0,0) *} A_{\mu}^{(0,0)} \\
H_{\mathrm{SL}}\left(\Omega_{\mathrm{LM}}{ }^{t}, \Omega_{\mathrm{MP}}{ }^{t}\right)=\sum_{\mu, m} F_{\mu, \mathrm{L}}^{(2, m) *}\left(\Omega_{\mathrm{LM}}{ }^{t}, \Omega_{\mathrm{MP}}{ }^{t}\right) A_{\mu, \mathrm{L}}^{(2, m)} \\
F_{\mu, \mathrm{L}}^{(2, m)}\left(\Omega_{\mathrm{LM}}{ }^{t}, \Omega_{\mathrm{MP}}{ }^{t}\right)=\sum_{m^{\prime} m^{\prime \prime}} D_{m m^{\prime \prime}}{ }^{2 *}\left(\Omega_{\mathrm{LM}}{ }^{t}\right) D_{m^{\prime \prime} m^{\prime}}{ }^{2 *}\left(\Omega_{\mathrm{MP}}{ }^{t}\right) F_{\mu, \mathrm{P}}^{\left(2, m^{\prime}\right)},
\end{gathered}
$$

where $F_{\mu, \eta}^{(2, m)}$ is proportional to the standard ISTO (irreducible spherical tensor operators), $\eta=\{\mathrm{L}, \mathrm{P}\}$ are the lab and principal references frames, respectively, $D^{2}$ are the Wigner rotation matrix elements of second rank, ${ }^{35} \Omega_{X Y}=\left(\gamma_{X Y}, \beta_{X Y}, \alpha_{X Y}\right)$ are the Euler angles for the $X \rightarrow Y$ transformation and $\mu$ is the type of interaction $g_{\mathrm{n}}, g, A_{i}$, i.e. nuclear-, electron-Zeeman and the hyperfine interaction for copper and ligand nuclei.

\section{A.1.1 Time scale separated Hamiltonian}

Concerning the time dependent Hamiltionian eqn (A3) we proceed to consider a time scale separation between the fast vibrations and restricted orientation motions causing the stochastic time dependence $\Omega_{\mathrm{MP}}(t)$ and the slower rotational diffusion $\Omega_{\mathrm{LM}}(t)$ fluctuations, see Fig. 1. We proceed with a "two step model" used in NMR relaxation studies ${ }^{36}$ and applied in previous SLEL work. ${ }^{34}$ With $\langle\cdot\rangle_{\mathrm{MP}}$ denoting a short time average over fast fluctuating $\Omega_{\mathrm{MP}}(t)$ we get the slow (s) component as

$$
\begin{aligned}
H_{\mathrm{SL}}^{(\mathrm{s})}\left(\Omega_{\mathrm{LM}}(t)\right)= & \left\langle H_{\mathrm{SL}}\left(\Omega_{\mathrm{LM}}(t), \Omega_{\mathrm{MP}}(t)\right)\right\rangle_{\mathrm{MP}} \\
= & \sum_{\mu, m} \sum_{m^{\prime} m^{\prime \prime}} D_{m m^{\prime \prime}}{ }^{2 *}\left(\Omega_{\mathrm{LM}}(t)\right) \\
& \times\left\langle D_{m^{\prime \prime} m^{\prime}}{ }^{2 *}\left(\Omega_{\mathrm{MP}}(t)\right)\right\rangle_{\mathrm{MP}} F_{\mu, \mathrm{P}}^{\left(2, m^{\prime}\right)} A_{\mu, \mathrm{L}}^{(2, m)}
\end{aligned}
$$

and the fast component as

$$
H_{\mathrm{SL}}^{(\mathrm{f})} \equiv H_{\mathrm{SL}}\left(\Omega_{\mathrm{LM}}(t), \Omega_{\mathrm{MP}}(t)\right)-H_{\mathrm{SL}}^{(\mathrm{s})}\left(\Omega_{\mathrm{LM}}(t)\right) .
$$

Furthermore, assuming fluctuations with at least three-fold symmetry in the (M) frame and cylindrical symmetric $F_{\mu, \mathrm{P}}^{(2, m \prime)}$ only $S^{\mathrm{MP}}=\left\langle D_{00}{ }^{2^{*}}\left(\Omega_{\mathrm{MP}}(t)\right)\right\rangle_{\mathrm{MP}}$, a second rank order parameter is non-zero and

$$
H_{\mathrm{SL}}^{(\mathrm{s})}\left(\Omega_{\mathrm{LM}}{ }^{t}\right)=\sum_{\mu, m} A_{\mu, \mathrm{L}}^{(2, m)} D_{m 0} 0^{2 *}\left(\Omega_{\mathrm{LM}}{ }^{t}\right) S^{\mathrm{MP}} F_{\mu, \mathrm{P}}^{(2,0)} .
$$

Provided the stochastic time dependence of $H_{\mathrm{SL}}^{(\mathrm{f})}$ is fast on the EPR timescale i.e. $\left\|\Delta H_{\mathrm{SL}}\right\| \tau_{\mathrm{f}} \ll 1$ (where $\tau_{\mathrm{f}}$ is the characteristic time scale for $\Omega_{\mathrm{MP}}$ fluctuations and $\left\|\Delta H_{\mathrm{SL}}\right\|$ is the anisotropy of the spin Hamiltonian), the $f$ part may treated using Redfield theory. ${ }^{8,9}$ The partial averaging has been treated for EPR problems by considering a nitroxide spin label in biomolecules. ${ }^{51,52}$ A general form of Liouvillian corresponding to fast processes in SLEL takes the form $\mathscr{L}^{(\mathrm{f})}\left(\Omega_{\mathrm{LM}}(t)\right)=-i \mathscr{R}^{(\mathrm{f})}\left(\Omega_{\mathrm{LM}}(t)\right)$ where $\mathscr{R}^{(\mathrm{f})}\left(\Omega_{\mathrm{LM}}(t)\right)$ is a Redfield matrix. It is interesting to note that there is a time dependence in the matrix originating from 
the orientation dependence in Redfield terms derived in ref. 51 and 52. The SLEL takes the form:

$$
\begin{aligned}
\frac{\mathrm{d} \rho\left(\Omega_{\mathrm{LM}}(t)\right)}{\mathrm{d} t}= & \left(-i\left[\mathscr{L}_{\mathrm{S}}+\mathscr{L}_{\mathrm{SL}}^{(\mathrm{s})}\left(\Omega_{\mathrm{LM}}(t)\right)\right]\right. \\
& \left.-\mathscr{R}^{(\mathrm{f})}\left(\Omega_{\mathrm{LM}}(t)\right)\right) \rho\left(\Omega_{\mathrm{LM}}(t)\right),
\end{aligned}
$$

and may be solved using the algorithm derived in ref. 11. However, we expect the fast dynamics to be in the extreme narrowing regime, $\omega_{0} \tau_{\mathrm{f}} \ll 1$ where $\omega_{0}$ is the resonance frequency and $\mathscr{R}^{(\mathrm{f})}$ to have only a very small influence on the observable. This motivates to use a Bloch form in treating residual line width. ${ }^{53}$ We solve the following SLEL in a matrix representation (see Appendix A.2)

$$
\frac{\mathrm{d} \rho^{(\mathrm{s})}\left(\Omega_{\left.\mathrm{LM}^{t}\right)}\right.}{\mathrm{d} t}=-i\left[L_{\mathrm{S}}+L_{\mathrm{SL}}^{(\mathrm{s})}\left(\Omega_{\mathrm{LM}^{t^{\prime}}}\right)\right] \rho^{(\mathrm{s})}\left(\Omega_{\mathrm{LM}}{ }^{t}\right),
$$

compute $\overline{\rho_{\mathrm{obs}}^{(\mathrm{s})}\left(\Omega_{\mathrm{LM}}{ }^{t}\right)}=\overline{\operatorname{Tr}\left\{S_{+}^{(1)} \rho^{(\mathrm{s})}\left(\Omega_{t}\right)\right\}}$ and finally compute the line shape with $\overline{\rho_{\text {obs }}\left(\Omega_{\mathrm{LM}^{t}}\right)}=\exp \left(-R_{2}^{(\mathrm{f})} t\right) \overline{\rho_{\text {obs }}^{(\mathrm{s})}\left(\Omega_{\mathrm{LM}}{ }^{t}\right)}$, where $R^{(\mathrm{f})}$ is a scalar. Comparing eqn (A8) and (A9), to separately solve for $\rho^{(\mathrm{s})}\left(\Omega_{\mathrm{LM}}{ }^{t}\right)$, eqn (A9) remains valid provided $R^{(\mathrm{f})} \ll\left\|H_{\mathrm{SL}}^{(\mathrm{s})}\left(\Omega_{\mathrm{LM}}{ }^{t}\right)\right\|$. From experimentally determined $R^{(\mathrm{f})}$ and $S^{\mathrm{MP}}$ an order of magnitude estimate of fast dynamics $\left(\tau_{\mathrm{f}}\right)$ may be computed under extreme narrowing conditions ${ }^{36}$ as

$$
R_{2}^{(\mathrm{f})}=5\left(1-\left(S^{\mathrm{MP}}\right)^{2}\right)\left[\left(F_{g, \mathrm{P}}^{(2,0)}\right)^{2}+\left(F_{A, \mathrm{P}}^{(2,0)}\right)^{2}+F_{g, \mathrm{P}}^{(2,0)} F_{A, \mathrm{P}}^{(2,0)}\right] \tau_{\mathrm{f}},
$$

including the dominating relaxation terms of $g$ and $\mathrm{Cu}$ (II) hyperfine anisotropy.

\section{A.1.2 Spin Hamiltonian elements}

The components of the irreducible electron Zeeman (magnetic) tensor in the principal frame $(\mathrm{P})$ are

$$
\begin{gathered}
F_{g}^{(0,0)}=-\sqrt{\frac{1}{3} \frac{2 \pi \beta_{\mathrm{e}}}{h}\left(g_{x x}+g_{y y}+g_{z z}\right),} \\
F_{g, \mathrm{P}}^{(2,0)}=\sqrt{\frac{2}{3}} \frac{2 \pi \beta_{\mathrm{e}}}{h}\left(g_{z z}-\frac{g_{x x}+g_{y y}}{2}\right),
\end{gathered}
$$

$$
\begin{gathered}
F_{g, \mathrm{P}}^{(2, \pm 1)}=0, \\
F_{g, \mathrm{P}}^{(2, \pm 2)}=\frac{1}{2} \frac{2 \pi \beta_{\mathrm{e}}}{h}\left(g_{x x}-g_{y y}\right),
\end{gathered}
$$

where $\beta_{\mathrm{e}}$ is the Bohr magneton. The Zeeman spin operators are

$$
\begin{aligned}
& A_{g, \mathrm{~L}}^{(0,0)}=-\sqrt{\frac{1}{3}}\left(B_{0} S_{z}\right), \\
& A_{g, \mathrm{~L}}^{(2,0)}=\sqrt{\frac{2}{3}}\left(B_{0} S_{z}\right), \\
& A_{g, \mathrm{~L}}^{(2, \pm 1)}=\mp \frac{1}{2}\left(B_{0} S_{ \pm}\right),
\end{aligned}
$$

$$
A_{g, \mathrm{~L}}^{(2, \pm 2)}=0
$$

where $S_{ \pm}$are combined from cartesian operators as $S_{ \pm}=S_{x} \pm$ $i S_{y}$. We consider only isotropic nuclear Zeeman interaction from, the $\mathrm{Cu}(\mathrm{II})$ nucleus with spin Hamiltonian

$$
F_{g_{\mathrm{n}}}^{(0,0)}=-\frac{2 \pi \mu_{\mathrm{n}}}{h} g_{\mathrm{n}}, A_{g_{\mathrm{n}}, \mathrm{L}}^{(0,0)}=B_{0} I_{z},
$$

where $\mu_{\mathrm{n}}, h, g_{\mathrm{n}}$ and $I_{\mathrm{z}}$ are the nuclear magneton, Planck's constant, isotropic chemical shift, and nuclear spin operator respectively. For hyperfine interaction the tensor operators are given by:

$$
\begin{gathered}
F_{A_{i}}^{(0,0)}=-\sqrt{\frac{1}{3} \frac{2 \pi \beta_{\mathrm{e}} g_{\mathrm{e}}}{h}}\left(A_{i x x}+A_{i y y}+A_{i z z}\right), \\
F_{A_{i}, \mathrm{P}}^{(2,0)}=\sqrt{\frac{2}{3} \frac{2 \pi \beta_{\mathrm{e}} g_{\mathrm{e}}}{h}}\left(A_{i z z}-\frac{A_{i y y}+A_{i z z}}{2}\right), \\
F_{A_{i}, \mathrm{P}}^{(2, \pm 1)}=0 \\
F_{A_{i}, \mathrm{P}}^{(2, \pm 2)}=\frac{1}{2} \frac{2 \pi \beta_{\mathrm{e}} g_{\mathrm{e}}}{h}\left(A_{i y y}-A_{i z z}\right),
\end{gathered}
$$

in the principal frame for nucleus $i$ and $g_{\mathrm{e}}$ is the free electron $g$ value. The corresponding spin operators are:

$$
\begin{gathered}
A_{A_{i}, \mathrm{~L}}^{(0,0)}=-\sqrt{\frac{1}{3}}\left(I_{i z} S_{z}+\frac{1}{2}\left(S_{+} I_{i-}+S_{-} I_{i+}\right)\right), \\
A_{A_{i}, \mathrm{~L}}^{(2,0)}=\sqrt{\frac{2}{3}}\left(I_{i z} S_{z}-\frac{1}{4}\left(S_{+} I_{i-}+S_{-} I_{i+}\right)\right), \\
A_{A_{i}, \mathrm{~L}}^{(2, \pm 1)}=\mp \frac{1}{2}\left(S_{ \pm} I_{i z}+S_{z} I_{i \pm}\right), \\
A_{A_{i}, \mathrm{~L}}^{(2, \pm 2)}=\frac{1}{2}\left(S_{ \pm} I_{i \pm}\right) .
\end{gathered}
$$

\section{A.2}

\section{A.2.1 Numerical procedure}

For numerical purposes, it is convenient to specify a basis and express eqn (A9) in matrix form. Using an orthonormal basis $\left[Q_{1}, Q_{2}, \ldots, Q_{M}\right]$, we get

$$
\begin{gathered}
\rho_{k l}=\rho_{i}=\left(Q_{i} \mid \rho\right), \\
L_{k l, m n}=L_{i j}=\left(Q_{i}|\mathscr{L}| Q_{j}\right),
\end{gathered}
$$

using the scalar product $(A \mid B) \equiv \operatorname{Tr}\left(A^{\dagger} B\right)$. In practice each of the matrices corresponding to the operator $A_{\mu, \mathrm{L}}^{(2, m)}[c f$. eqn (A7)] are pre-computed in the matrix using Spindynamica software. ${ }^{54}$ The matrices are loaded from files and multiplied with time the dependent scalar functions $F_{\mu, \mathrm{L}}^{l, m}\left(\Omega_{\mathrm{LM}}(t)\right)$. For the six interactions, electron Zeeman, $\mathrm{Cu}$ (II) hyperfine and four ${ }^{14} \mathrm{~N}$ ligand hyperfine, this corresponds to 36 Liouville matrices each of dimension $M=419904$.

The observable over the the time window $[0, T]$ is computed as summarized in the following steps: 

$P\left(\Omega_{0}\right)$

(2) Simulate a Brownian trajectory on sphere ${ }^{37,55}$ and compute $\left(\Omega_{n}\right)$ at $n=0,1, \ldots, N$.

(3) Numerically solve eqn (A9) ${ }^{11}$ for $\tilde{\rho}_{\text {obs }}\left(\Omega_{n}\right)$ at $n=0,1, \ldots, N$.

(4) Repeat steps 1-3 $L$ times and compute $\mathscr{A}\left(\tilde{\rho}_{\text {obs }}\left(\Omega_{n}\right), L\right)$ as follows:

In summary, the solution is calculated $L$ times, where $L$ is chosen such that the statistical error in the observable is sufficiently small. An implicit numerical scheme implemented in C-code is found to be particularly efficient ${ }^{11}$ in combination with the PETSc library ${ }^{56-58}$ for matrix operations.

\section{Acknowledgements}

Grant sponsor: United Kingdom Engineering and Physical Sciences Research Council; contract/grant number: EP/ F006802/1. The authors acknowledge the use of the IRIDIS High Performance Computing Facility, and associated support services at the University of Southampton, in the completion of this work. The EPSRC UK national service for EPR in Manchester is acknowledged for providing spectrometers and Professor David Collison for advice in performing the EPR experiments.

\section{References}

1 F. I. Rosell, M. R. Mauk and A. G. Mauk, Biochemistry, 2005, 44, 1872-1979.

2 R. Basosi, G. D. Lunga and R. Pogni, in Biomedical EPR-Part A: Free radicals, metals, medicine and Physiology, ed. S. R. Eaton, G. R. Eaton and L. J. Berliner, Kluwer Academic/Plenum Publishers, New York, 2005, ch. 13, pp. 385416.

3 W. W. E. Antholine, in Biomedical EPR-Part A: Free radicals, metals, medicine and Physiology, ed. S. R. Eaton, G. R. Eaton and L. J. Berliner, Kluwer Academic/Plenum Publishers, New York, 2005, ch. 14, pp. 417-454.

4 J. S. Hyde, B. Bennett, E. D. Walter, G. L. Millhauser, J. W. Sidabras and W. E. Antholine, Biophys. J., 2009, 96, 3354-3362.

455 J. Leigh and G. Reed, J. Phys. Chem., 1971, 75, 1202-1204.

6 J. S. Hyde and W. Froncisz, Annu. Rev. Biophys. Bioeng., 1982, 11, 391-417.

7 M. Kaupp, M. Bühl and V. G. Malkin, Calculation of NMR and EPR Parameters, Wiley-VCH, Weinheim, 2004.

508 A. G. Redfield, Adv. Magn. Reson., 1965, 1, 1-32.

9 J. H. Freed and G. K. Fraenkel, J. Chem. Phys., 1963, 39, 326348.

10 K. V. S. Rao, C. F. Polnaszek and J. H. Freed, J. Phys. Chem., 1977, 81, 449-456.

11 P. Håkansson and P. B. Nair, Phys. Chem. Chem. Phys., 2011, 13, 9578-9589.
12 R. Neiman and D. Kivelson, J. Chem. Phys., 1961, 35, 156161.

13 A. Schweiger and G. Jeschke, Principles of pulse electron paramagnetic resonance, Oxford University Press, 2001.

14 W. Froncisz and J. S. Hyde, J. Chem. Phys., 1980, 73, 31233131.

15 S. Stoll and A. Schweiger, J. Magn. Reson., 2006, 178, 42-55.

16 G. R. Hanson, K. E. Gates, C. J. Noble, M. Griffin, A. Mitchell and S. Benson, J. Inorg. Biochem., 2004, 98, 903-916.

17 E. Aronoff-Spencer, C. S. Burns, N. I. Avdievich, G. J. Gerfen, J. Peisach, W. E. Antholine, H. L. Ball, F. E. Cohen, S. B. Prusiner and G. L. Millhauser, Biochemistry, 2000, 39, 13760-13771.

18 E. Meirovitch, D. Igner, E. Igner, G. Moro and J. H. Freed, J. Chem. Phys., 1982, 77, 3915-3938.

19 K. A. Earle and D. E. Budil, Advanced ESR methods in Polymer research, John Wiley \& Sons, Inc., 2006.

20 R. F. Campbell and J. H. Freed, J. Phys. Chem., 1980, 84, 2668-2680.

21 J. W. Chen, F. P. Auteri, D. E. Budil, R. L. Belford and R. B. Clarkson, J. Phys. Chem., 1994, 98, 13452-13459.

22 D. Kruk, J. Kowalewski, D. S. Tipikin, J. H. Freed, M. Mościcki, A. Mielczarek and M. Port, J. Chem. Phys., 2011, 134, 024508.

23 G. D. Lunga, R. Pogni and R. Basosi, J. Phys. Chem., 1994, 98, 3937-3942.

24 M. Pasenkiewicz-Gierula, W. Subczynski and W. Antholine, J. Phys. Chem. B, 1997, 101, 5596-5606.

25 G. D. Lunga, M. Pezzato, M. Camilla Baratto, R. Pogni and R. Basosi, J. Magn. Reson., 2003, 164, 71-77.

26 H. Eviatar, E. van Fassen and Y. Levine, Chem. Phys. Lett., 1992, 195, 233-238.

27 B. Robinson, L. Slutsky and F. Auteri, J. Chem. Phys., 1992, 96, 2609.

28 N. Usova, P.-O. Westlund and I. Fedchenia, J. Chem. Phys., 1995, 103, 96-103.

29 P. Håkansson, P.-O. Westlund, E. Lindahl and O. Edholm, Phys. Chem. Chem. Phys., 2001, 3, 5311.

30 D. P. Rangel, P. C. Baveye and B. Robinson, J. Phys. Chem. B, 2012, 116, 6233-6249.

31 D. Sezer, J. H. Freed and B. Roux, J. Chem. Phys., 2008, 128, 165106.

32 P. Håkansson and M. Mella, J. Chem. Phys., 2007, 126, 104106.

33 A. Keane and P. Nair, Computational Approaches for Aerospace Design: The Pursuit of Excellence, Wiley, 2005.

34 K. Åman, P. Håkansson and P.-O. Westlund, Phys. Chem. Chem. Phys., 2005, 7, 1394-1401.

35 D. M. Brink and G. R. Satchler, Angular Momentum, Oxford University Press, 1993.

36 B. Halle and H. Wennerström, J. Chem. Phys., 1981, 75, 1928.

37 P. Håkansson, L. Persson and P.-O. Westlund, J. Chem. Phys., 2002, 117, 8634-8643.

38 E. Stulz, S. M. Scott, Y.-F. Ng, A. D. Bond, S. J. Teat, S. L. Darling, N. Feeder and J. K. M. Sanders, Inorg. Chem., 2003, 42, 6564-6574.
1

10 
39 K. Khairy, D. Budil and P. Fajer, J. Magn. Reson., 2006, 183, 152-159.

40 G. D. Lunga, R. Pogni and R. Basosi, Mol. Phys., 1998, 95, 1275-1281.

41 T. Spatek, P. Pietrzyk and Z. Sojka, J. Chem. Inf. Model., 2005, 45, 18-29.

42 J. A. Nelder and R. Mead, Comput. J., 1965, 7, 308-313.

43 V. G. Shtyrlin, Y. I. Zyavkina, E. M. Gilyazetdinov, M. S. Bukharov, A. A. Krutikov, R. R. Garipov, A. S. Mukhtarov and A. V. Zakharov, Dalton Trans., 2012, 41, 1216-1228.

44 C. Finazzo, C. Calle, S. Stoll, S. Van Doorslaer and A. Schweiger, Phys. Chem. Chem. Phys., 2006, 8, 1942-1953. 45 J. A. Shelnutt, X.-Z. Song, J.-G. Ma, S.-L. Jia, W. Jentzen, C. J. Medforth and C. J. Medforth, Chem. Soc. Rev., 1998, 27, 31-42.

46 C. Medforth, R. Haddad, C. Muzzi, N. Dooley, L. Jaquinod, D. Shyr, D. Nurco, M. Olmstead, K. Smith, J. Ma and J. Shelnutt, Inorg. Chem., 2003, 42, 2227-2241.

47 P.-O. Westlund and H. Wennerström, Phys. Chem. Chem. Phys., 2012, 12, 201-206.

48 J. H. Kim, S. H. Lee, J. S. Lee, M. Lee and C. B. Park, Chem. Commun., 2011, 47, 10227-10229.

49 S. V. Doorslaer, I. Caretti, I. Fallis and D. Murphy, Coord. Chem. Rev., 2009, 253, 2116-2130.
50 P. L. Nordio, General Magnetic Resonance Theory, Academic Press, Inc., 1976, ch. 2, pp. 5-52.

51 R. Cassol, A. Ferrarini and P. L. Nordio, J. Phys. Chem., 1993, 97, 2933-2940.

52 F. Tombolato, A. Ferrarini and J. H. Freed, J. Phys. Chem. B, 2006, 110, 26260-26271.

53 D. Gamliei and H. Levanon, Stochastic Processes in Magnetic Resonance, World Scientific Publishing Co. Pte. Ltd., 1995.

54 Some of the work in this article employed the SpinDynamica code for Mathematica, programmed by Malcolm H. Levitt, available at www.SpinDynamica.soton.ac.uk.

55 P. Håkansson, M. Isaksson, P.-O. Westlund and L. B.-Å. Johansson, J. Phys. Chem. B, 2004, 108, 17243-17250.

56 S. Balay, K. Buschelman, W. D. Gropp, D. Kaushik, M. G. Knepley, L. C. McInnes, B. F. Smith and H. Zhang, PETSc Web page, 2009, http://www.mcs.anl.gov/petsc.

57 S. Balay, K. Buschelman, V. Eijkhout, W. D. Gropp, D. Kaushik, M. G. Knepley, L. C. McInnes, B. F. Smith and H. Zhang, PETSc Users Manual, Argonne National Laboratory Technical Report ANL-95/11 - Revision 3.0.0, 2008.

58 S. Balay, W. D. Gropp, L. C. McInnes and B. F. Smith, Modern Software Tools in Scientific Computing, 1997, pp. 163-202. 